\title{
Effect of solvent choice on breath-figure-templated assembly of "holey" polymer films
}

\author{
Vivek Sharma ${ }^{1(a)}$, Lulu Song ${ }^{1}$, Richard L. Jones ${ }^{3}$, Matthew S. Barrow $^{3}$, P. Rhodri Williams ${ }^{3}$ \\ and Mohan Srinivasarao ${ }^{1,2(b)}$ \\ ${ }^{1}$ School of Materials Science Engineering, Georgia Institute of Technology - Atlanta, GA, USA \\ ${ }^{2}$ School of Chemistry and Biochemisty, Georgia Institute of Technology - Atlanta, GA, USA \\ ${ }^{3}$ School of Engineering, Swansea University - Swansea, UK, EU
}

received on 4 July 2010; accepted by S. Kumar on 20 July 2010

published online 5 August 2010

PACS 83.80.Rs - Polymer solutions

\begin{abstract}
Dew or breath figures is a disordered pattern formed by polydisperse water drops that form when a cold surface comes in contact with breath or moist air. Unexpectedly, self-assembled arrays of non-coalescent monodisperse water drops form when dilute solutions of polymers in volatile organic solvents are exposed to moist air. After solvent evaporation is complete, these condensation figures dry out as well, leaving "holey" polymer films, containing hexagonally ordered arrays of pores $(0.2$ to $10 \mu \mathrm{m})$. While the macroporous films are produced easily for a wide variety of solvents and polymers, there is no existing theory that describes how the pore size depends upon tunable parameters like humidity, air temperature and velocity as well as on the choice of solvent and polymer. In this study, we propose a transport model to elucidate the role of the solvent and airflow in determining the rate and extent of evaporative cooling, and contrast our model results with the corresponding experimental measurements for polystyrene/carbon disulfide solutions. We describe how modeling evaporative cooling is essential for the quantitative understanding of dominant processes that lead to growth, non-coalescence and self-assembly of water drops, and the subsequent formation of ordered arrays of pores.
\end{abstract}

editor's choice Copyright (C) EPLA, 2010

Breath figures or dew forms over cold solid or liquid substrates on contact with humid air [1-5]. The growing water drops coalesce with each other, and several generations of drops coexist in a fractal pattern. When the breath figures form over a pre-cooled liquid substrate (immiscible with water), they first self-organize into a locally ordered state, and then coalescence sets in, giving a pattern with polydisperse drop sizes [3-5]. Breath-figure-like patterns of water droplets appear on an evaporating polymer solution, exposed to a stream of moist air, and once the solvent evaporation is complete, the water drops evaporate away as well, leaving a highly ordered array of holes in the polymer film [6]. To name the process as breath-figure-templated assembly is to recognize growing amount of experimental evidence [6-8] showing that the formation of pores occurs as a result of nucleation, growth and self-assembly of non-coalescent water drops over evaporatively cooled polymer solution, as postulated

\footnotetext{
(a) Current address: Hatsopoulos Microfluids Laboratory, Department of Mechanical Engineering, Massachusetts Institute of Technology - Cambridge, MA, USA.

(b) E-mail: mohan@ptfe.gatech.edu
}

by Srinivasarao and coworkers [6]. The templating process has been successfully applied to a wide variety of polymers (coil-like, rod-coil, star-shaped) and solvents (chloroform, toluene, carbon disulfide, dichloromethane, benzene, THF) and the holey films are developed for applications such as non-wetting or superhydrophobic surfaces, picoliter beakers, microlenses, substrates for cell growth and photovoltaics, etc. $[6,7,9-15]$. Since applications require microporous films with a well-defined structure, we need to determine how the pore size depends upon the parameters like humidity, air temperature and velocity as well as the choice of solvent and polymer.

Despite a general consensus that the fabrication process is simple to perform [6,7,9-15], models capable of quantitatively predicting the final structure in terms of these aforementioned variables are not yet available, partially due to the complex interplay of several physical processes [6]. The problem has been cited to be very complex $[6,10,12]$, not only because it requires an understanding of mass and heat changes taking place during the solvent evaporation, coupled with the kinetics of growth and assembly of water drops, but possibly because 
it also involves convection within the polymer solution substrate, and the presence of non-coalescence in water drops. Local variations in the number density of growing drops as well as local variations in transport fluxes are likely to occur. The temperature and/or concentration gradient induced phenomena of Rayleigh-Bénard and/or Marangoni-Bénard convection $[6,8,16,17]$ could become important during the templating process. It is clear, however, that both heat and mass transfer are predominantly influenced by the choice of solvent and airflow conditions, and it must be therefore useful in the first instance to examine the correlation between solvent properties and evaporative cooling.

In this paper, we describe a model for computing the concentration and temperature changes occurring in the polymer solution during the breath-figure-templated assembly (BFTA) of ordered arrays of pores. We show that the diffusion-based model captures the experimentally observed behavior for a PS/CS 2 system, and thus we establish a quantitative methodology for contrasting the primary effect of the choice of a solvent. Before describing the model calculations and experimental results, we explain how our present study is the necessary step in elucidating the physical processes and parametric space underlying the breath-figure-templated assembly of pores in polymer films.

The templating mechanism for the formation of microporous films in our case is driven by the evaporation of a high-volatility solvent (aided by a flow of moist air over the solution), and the evaporative cooling drives the nucleation and growth of water droplets. It has been suggested by Limaye et al. [8] and Srinivasarao et al. [6] that evaporative cooling induces convection currents within the solution which, in conjunction with the airflow across the surface, drive ordering of the water droplets. The noncoalescence of water drops has been attributed to several factors although the dominant mechanism is currently being studied. Srinivasarao et al. [6] suggested that the temperature gradient present in the system may produce thermocapillary convection such that the droplets are kept apart by replenishing the fluid (air) layer that needs to be drained for drops to come together [18]. An alternative hypothesis is that evaporation flux may be providing a non-vanishing, replenished vapor layer to keep drops from coalescing [6]. We derive both the rate at which vapor flux is generated and how the temperature above the solution changes as a function of time, and their role in noncoalescence and self-assembly will be described in detail elsewhere [19].

The nucleation and growth of droplets on solid substrates has been studied extensively in the context of the formation of dew and breath figures [2-5], as well as in developing a greater understanding of chemical vapor deposition [20]. In those experiments and models [2-5], the systems considered differ from polymer solutions in at least two important aspects: 1) the substrate is pre-cooled below the dew point and 2) coalescence is the dominant growth mechanism in the late stages. In polymer solutions, cooling is evaporation driven, the drops can remain non-coalescent and these grow and assemble into a close-packed, highly ordered array of similar size drops (packing fraction approaching 0.90). The significant difference in growth kinetics and patterns formed necessitates the quantitative analysis of factors underlying droplet growth and assembly over evaporating polymer solutions.

In the present study, we first compare the experimentally determined mass loss due to evaporation with a model that considers the diffusion of solvent through a polymer film with airflow at the top surface. A thin film of polymer solution, with polymer content $C(C<5 \%)$ is deposited on a solid substrate. The film is initially at the ambient temperature, $T$. Let us assume that the solution is spread into a rectangular block of area, $S$, with initial height $h_{0}$ and initial volume $V_{0}$. The presence of the polymer retains the boundary of the film, thus maintaining the area of the film constant, while the volume of the fluid decreases with time. A flow of humidified air, with velocity $v_{\infty}$ and kinematic viscosity $v_{a}$ is considered to move in a direction $x$ such that a laminar boundary layer is formed close to the surface of the polymer solution according to the Reynolds number condition $\left(R e \sim v_{\infty} x / v_{a}>10^{5}\right)$ for a boundary layer flow which presently corresponds to velocities $v_{\infty} \sim 1 \mathrm{~m} / \mathrm{s}$ (typical velocities from experiments).

Let us consider the evaporative mass loss problem by proposing that the liquid solvent diffuses through the polymer solution to arrive at the surface, where it is removed by the blast of moist air. The problem is therefore considered to be predominantly diffusion limited and may be conveniently described by Fick's law, such that the diffusion process within the polymer solution is represented by

$$
D_{L} \frac{\partial^{2} C_{L}}{\partial z^{2}}=\frac{\partial C_{L}}{\partial t}
$$

where $D_{L}$ is the diffusion coefficient of the solvent, and $C_{L}$ is the concentration of the solvent at position $z$ in the film. At the bottom surface of the film, no solvent loss takes place through the supporting substrate and hence the boundary condition at this surface

$$
\frac{\partial C_{L}}{\partial z}=0 \text { at } z=0 \forall t
$$

As the sample is sufficiently thin, mass loss is considered to occur only at the top surface due to the negligible area for mass transfer presented by the sample edges. At the top surface, the solvent is considered to be continuously removed by mass transfer to the airflow, and hence the appropriate boundary condition is

$$
\frac{\partial C_{L}}{\partial z}=\frac{k_{M T}}{D_{L} K}\left(C_{L}^{*}-C_{L}\right) \text { at } z=l, t>0 .
$$

$K$ is the equilibrium distribution coefficient and $C_{L}^{*}=$ $K C_{L \infty}$. In effect, we use the Henry law, and the term 


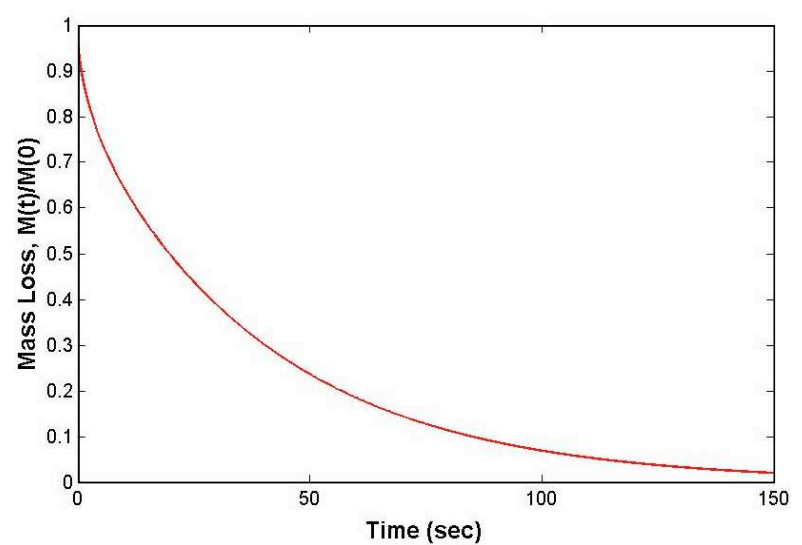

Fig. 1: (Colour on-line) Theoretically predicted mass loss as a function of time for PS/ $\mathrm{CS}_{2}$, using a flow rate of $0.75 \mathrm{~m} / \mathrm{s}$, and a thickness corresponding to $0.12 \mathrm{~g}$ of the film, area $1 \mathrm{~cm}^{2}$ (typical experimental values).

$K C_{L \infty}$ is replaced by the concentration inside the film substrate in equilibrium with the bulk concentration in vapor $[21,22]$. The mass transfer coefficient, $k_{M T}$, for the transfer of component $\mathrm{A}$ into the free stream of $\mathrm{B}$ is defined in terms of the diffusion at the interface as

$$
k_{M T}=-\frac{\left.D_{L}\left(\partial C_{L} / \partial z\right)\right|_{z=0}}{C_{L 1}-C_{L \infty}} .
$$

For laminar flow, the mass transfer coefficient can be estimated from the Sherwood number, $S h_{\text {LAMINAR }}=$ $k_{M T} l / D_{L}=0.664 R e^{1 / 2} S c^{1 / 3}$. Here the Schmidt number, $S c=\nu / D$ is the ratio of momentum diffusivity to mass diffusivity [22], and for a typical case of $1 \mathrm{~m} / \mathrm{s}$ moist airflow over a carbon disulfide solution, $\operatorname{Re}(\sim 1000)$ and $S c(=1.2)$ correspond to measures with respect to air diffusivity, viscosity and velocity. The mass transfer coefficient for the turbulent flow has a different correlation [22].

The diffusion equation with the aforementioned boundary and initial conditions has a solution of the form

$$
\frac{M_{t}}{M_{0}}=\sum \frac{2 \alpha^{2}}{\left(\beta_{i}^{2}+\alpha^{2}+\alpha\right)} \exp \left(-\frac{\beta_{i}^{2} D_{L} t}{l^{2}}\right),
$$

where $\beta_{n}$ values are roots of $\beta_{n} \tan \beta_{n}=\alpha=k_{M T} l /$ $K D_{L}$ [21] and $M_{t} / M_{0}$ denotes the mass of the polymer solution relative to the initial mass as a function of time. The transient depletion of the solvent calculated above depends upon the velocity of airflow and solvent properties, as the values of mass transfer coefficient (and hence that of coefficients $\alpha$ and $\beta$ ) depend on these experimental parameters. The rate of the solvent loss is mainly limited by the diffusion coefficient of the solvent. Figure 1 shows the calculated mass loss as a function of time, and fig. 2 shows the corresponding experimentally measured evaporation profile for a $\mathrm{PS} / \mathrm{CS}_{2}$ solution (adapted from experiments done by Song [12]). The calculated rate of mass loss appears to closely resemble



Fig. 2: (Colour on-line) Experimentally determined mass profiles, for PS/ $\mathrm{CS}_{2}$ solutions, for polystyrene (mono-carboxy terminated, $M_{w}=50 \mathrm{~K}$ ) and airflow of $0.75 \mathrm{~m} / \mathrm{s}$, varied PS content.

the experimentally measured profiles with the exception of the more dilute solutions, where the surface area itself was not constant. The higher the initial concentration of polymer in the solvent, the better and sooner the contact line is pinned. The magnitude of $\beta$ is quite insensitive to change in velocity (for typical airflows employed; $0.25-1$ $\mathrm{m} / \mathrm{s}$ ) and the model computed mass loss occurs nearly at the same rate, in agreement with the experimental results of Song [12]. It is inherently assumed in the model that the choice of polymer and the molecular weight do not affect the time required for mass loss and studies conducted to date on a range of polymers used to form breath figures appear to support the validity of this assumption [12].

Next, we model the change in the temperature of the surface during evaporation, and compare it with experimental measurements. The variation of surface temperature as a function of time during the evaporative period is essentially a heat diffusion problem and may be described by the equation

$$
D_{T} \frac{\partial^{2} \theta}{\partial z^{2}}=\frac{\partial \theta}{\partial t} .
$$

We consider the polymer solution to be thin enough such that the dominant heat exchange takes place in the $z$-direction, and that the heat of mixing of solvent vapor and moist air is negligible. Let us define $\theta$ as the difference between the temperature of the solvent and the temperature of the airflow, $D_{T}$ is the thermal diffusion coefficient, given by $D_{T}=K_{T} / \rho_{L} \alpha_{L}^{\prime}, K_{T}$ is the heat conductivity and $\alpha_{L}^{\prime}$ is the specific heat of the solvent.

At the bottom surface, which is in contact with a solid insulating substrate, the boundary condition of zero flux is applied, implying

$$
\partial \theta / \partial z=0 \quad \text { at } \quad z=0 \quad \forall t .
$$

Heat is continuously exchanged at the top surface. The heat exchange associated with the enthalpy of evaporation 


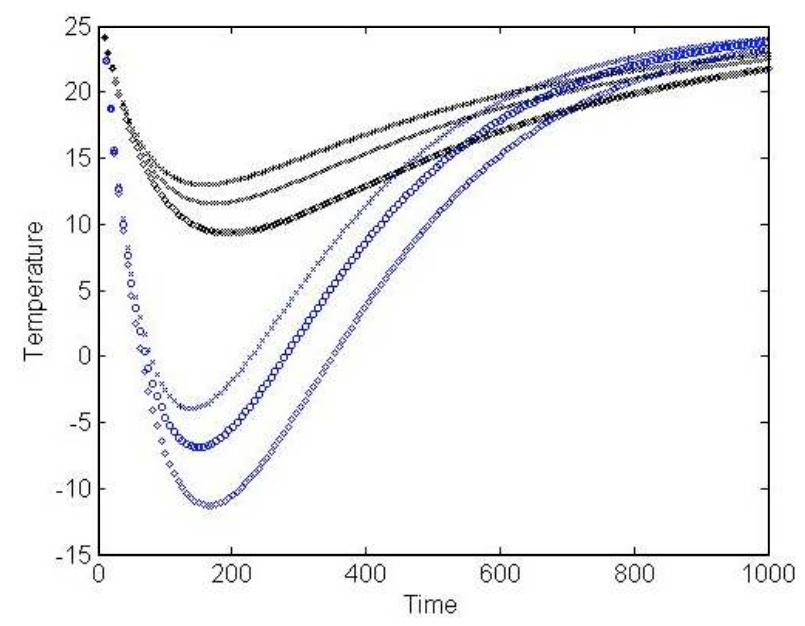

Fig. 3: (Colour on-line) Comparison of the model profiles of the temperature variation (in ${ }^{\circ} \mathrm{C}$ ) as a function of time (in seconds) plotted for carbon disulfide, $\mathrm{CS}_{2}$ (blue) and chloroform, $\mathrm{CHCl}_{3}$ (black) for flow rates of 0.5 (squares), 0.75 (circles) and 1 (crosses) $\mathrm{m} / \mathrm{s}$, respectively.

is $\frac{1}{2} \rho_{L} l L_{\text {vap }}(\mathrm{d} \mathfrak{R}(t) / \mathrm{d} t)$, where $\mathfrak{R}(t)=M(t) / M(0)$ is the relative mass at time $t$ and $L_{v a p}$ is the heat of vaporization. Radiative heat loss is equal to $k_{1} \theta$, where $k_{1}=$ $4 \sigma_{0} \theta_{0}^{3}$, where $\sigma_{0}$ is Stephan's constant. Heat loss due to airflow is equal to $k_{H T} \theta$, where $k_{H T}$ is dependent upon both the Reynolds number $R e$ and the Prandtl number $\operatorname{Pr}$ and is calculated using the Nusselt number, $N u_{L}=$ $k_{H T} L_{v a p} / K_{T}=0.664 \operatorname{Re}^{1 / 2} \operatorname{Pr}^{1 / 3}$. Lastly, conductive heat exchange is equal to $K_{T} \frac{\partial \theta}{\partial z}$. All heat exchange terms are incorporated in the boundary condition:

$$
\frac{1}{2} \rho_{L} l L_{v a p} \frac{\mathrm{d} \Re}{\mathrm{d} t}+K_{T} \frac{\partial \theta}{\partial z}-k_{H T} \theta=0 \text { at } z=l, \quad t>0 .
$$

The $k_{1}$ is dropped as the term is much smaller than the heat transfer coefficient, $k_{H T}$. By defining $\phi=\theta / \theta^{\prime}$; $\theta^{\prime}=\beta^{2} D_{L} \rho_{L} L_{v a p} \xi / 2 K_{T}, \quad X=z / l, \quad \tau=t / \tau^{\prime} ; \quad \tau^{\prime}=l^{2} / D_{T}$ (heat diffusion timescale), $\xi$ as the pre-factor for $M(t) / M(0), \quad \chi=k_{H T} l / D_{T} \alpha_{L}^{\prime} \rho_{L}$ (ratio of convective to diffusive heat transfer) and $\gamma=\beta^{2} D_{L} / D_{T}$, (related to the ratio of heat and mass diffusion timescales) the heat equation becomes

$$
\frac{\partial \phi}{\partial \tau}=\frac{\partial^{2} \phi}{\partial X^{2}}
$$

with the initial condition and the boundary conditions as

$$
\begin{aligned}
& \phi=0 ; \quad 0<X<1 ; \quad \tau=0 \\
& \frac{\partial \phi}{\partial X}=0 \text { at } X=0 \\
& \frac{\partial \phi}{\partial X}-\chi \phi+\exp (-\gamma \tau)=0 \text { at } X=1, \tau>0 .
\end{aligned}
$$

The heat equation, with the aforementioned boundary and initial conditions, was solved numerically for the



Fig. 4: (Colour on-line) Experimentally measured temperature profile, for a flow rate of $0.5 \mathrm{~m} / \mathrm{s}$, humidity $52 \%$, PS $3 \%$ by weight in $\mathrm{CS}_{2}$.

$\mathrm{PS} / \mathrm{CS}_{2}$ and $\mathrm{PS} / \mathrm{CHCl}_{3}$ system and the computed temperature as a function of time is shown in fig. 3. This shows a remarkable similarity to the corresponding experimentally measured temperature profile plotted in fig. 4 , which was obtained by using an embedded thermocouple probe, see SEM image in fig. 5 . We note that the temperature first decreases below room temperature and as the evaporation rate of the solvent slows down, the temperature starts to rise back again. The magnitude of the temperature drop and its duration appears to be controlled by the magnitude of thermal and mass diffusivity of the solvent (through value of $\gamma$ ) and by the convective losses as incorporated in the magnitude of the heat transfer coefficient, $k_{H T}$ (and hence the value of $\chi$ ).

If we contrast the temperature profiles for chloroform and carbon disulfide, fig. 3, for the flow rates shown, the extent of cooling is always larger for carbon disulfide. The difference in the extent of cooling is demonstrated remarkably well by a drop of carbon disulfide solution that clouds much faster in ambient conditions. Interestingly, a higher airflow velocity produces a smaller temperature change. The explanation is simple and as follows. Evaporative cooling is counteracted by an increased and opposing conductive heat flux, from the warmer air that flows over the colder substrate. By changing the depth and width of the curve that represents the variation in temperature with time, the time available for drops to nucleate and grow must change.

The kinetics of droplet nucleation, growth and assembly are determined by the mass and heat flux of water vapor and these are directly influenced by the local conditions controlled by the solvent evaporation. Specifically, we note that the water droplets are formed by a condensation process, which occurs when the temperature decreases below the critical dew point temperature in the vicinity of the substrate. The growth of droplets due to condensation 

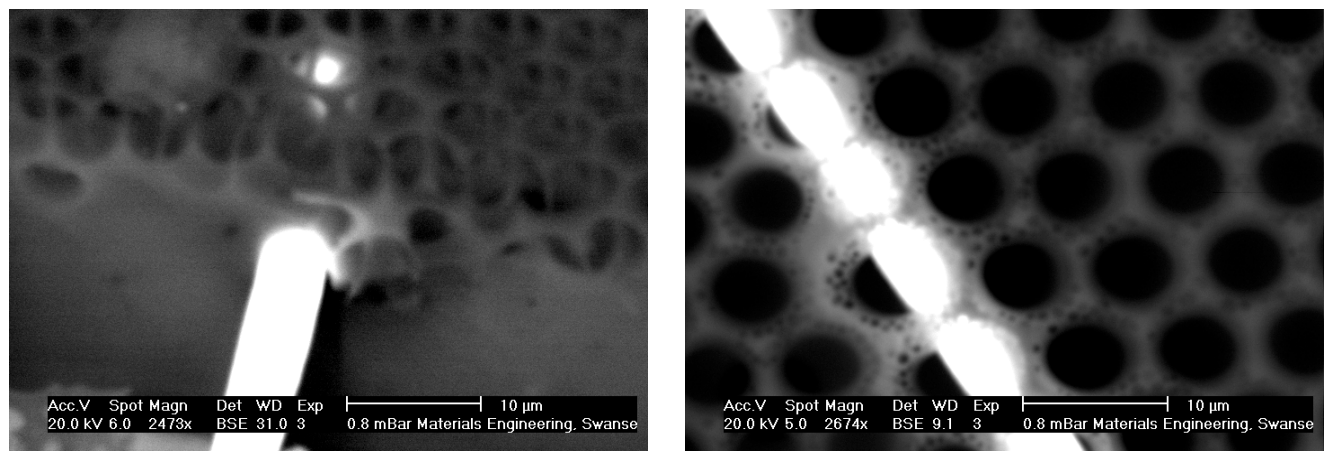

Fig. 5: SEM images for microporous films with an embedded wire (PS/CS 2 ), the scale bar is $10 \mu \mathrm{m}$.

is fundamentally limited to the period within which the polymer solution is maintained below the dew point. It is proposed that the transient temperature profile of the solution may be approximated by the singular cooling effect of evaporative solvent flux, thereby providing a simple and effective means for estimating the potential drop growth time and hence the eventual pore size. But even without formulating a growth law, one can expect that the increase in velocity implies lower drop sizes, for the drops can grow only for a shorter duration. This is in accord with the experimental observations reported by various groups $[6,23,24]$. By using the corresponding physical properties in our numerical solution, we can predict the temperature changes for any given solvent.

It is worth noting that as the temperature of the solution and the polymer concentration change, the absolute values of the physical properties of the solvent will vary accordingly. Also heat change accompanies the condensation and the evaporation of water drops. Even though these effects can be included explicitly, we estimated that the variation of the key parameters (including vapor pressure, heat of vaporization, specific heat and diffusivity of the liquid) is sufficiently low (i.e. within a few percent of those of the pure solvent) to justify their omission in the present treatment. Despite this and even with our simplifying approximations, the model appears to capture most of the aspects of the evaporation rate and cooling occurring within the context of breath-figure-templated assembly.

Knowing the polymer concentration and substrate temperature as a function of time provides an estimate of how the surface tension and viscosity of the solution changes during the droplet growth and assembly phases. As the polymer solutions progress from a dilute to semidilute state during evaporation, the associated viscosity increases by approximately three orders of magnitude [25]. In breath figures formed in liquids, the delayed coalescence of drops $[3,26]$ which allows for their assembly into rafts, is attributed to the draining time required for the solvent to move out which scales linearly with viscosity. In the late stages, where droplets are closely packed and lie within the polymer solution, the draining time for the increasingly viscous polymer solution will be at least three to four orders of magnitude larger than that of the pure solvent. The mechanism for non-coalescence during the early stages will be detailed elsewhere [19]. We infer that the assembly and pore size observed on varying architecture, molecular weight and concentration [6,12,27,28], (keeping the polymer solvent and airflow conditions the same), are related to how these factors influence the rate of change of viscosity of the underlying substrate. For drops to grow and self-assemble, the viscosity needs to vary at a rate that allows drops the mobility to evolve into these closed-packed arrays. The change in concentration and molecular weight directly influence the viscosity encountered by drops, and hence they influence the final morphology of the polymer films.

In this study, we show how the choice of solvent determines the rate and extent of evaporative cooling. The rate of mass loss and temperature change measured for a polymer solution, $\mathrm{PS} / \mathrm{CS}_{2}$ system, exposed to a stream of (moist) air are captured quite well by our model. We contrasted the extent of evaporative cooling for carbon disulfide and chloroform and determined that $\mathrm{CS}_{2}$ solutions have a greater temperature drop. The approach can be easily extended to other solvents, and polymer solutions. Our elucidation of how the choice of solvent and airflow velocity determines the timescales as well as heat and mass fluxes is an essential step towards our goal of unraveling the physics of nucleation, growth, non-coalescence and assembly of water drops and as a result, establishing how pore sizes can be manipulated using process parameters. The detailed model for kinetics of drop nucleation and growth will be described in a subsequent publication [19]. We note here that the rate of solvent evaporation and the associated concentration and temperature-dependent properties of a polymer solution affect the morphology obtained in a range of other systems including functional adhesives, porous membranes and anti-reflection coatings [29,30], fibers spun from solution (for example in electrospinning [31]) and 
spin casting [32]. Changes in concentration and temperature influence the kinetics and dynamics of phase transitions and, thus, they are of importance in processes such as the casting of immiscible polymer blends and block copolymers [33].

MS acknowledges financial support for this work from the National Science Foundation, through the Materials World Network Award, DMR-0603026 and through the Petroleum Research Foundation (PRF-Type AC).

\section{REFERENCES}

[1] Rayleigh, Nature, 86 (1911) 416

[2] Beysens D. and Knobler C. M., Phys. Rev. Lett., 57 (1986) 1433.

[3] Steyer A., Guenoun P., Beysens D. and Knobler C. M., Phys. Rev. B, 42 (1990) 1086.

[4] Beysens D., Steyer A., Guenoun P., Fritter D. and Knobler C. M., Phase Transitions, 31 (1991) 219.

[5] Beysens D., Atmos. Res., 39 (1995) 215.

[6] Srinivasarao M., Collings D., Philips A. and Patel S., Science, 292 (2001) 79.

[7] Barrow M. S., Jones R. L., Park J. O., Srinivasarao M., Williams P. R. and Wright C. J., Spectrosc.: Int. J., 18 (2004) 577.

[8] Limaye A. V., Narhe R. D., Dhote A. M. and Ogale S. B., Phys. Rev. Lett., 76 (1996) 3762.

[9] Widawski G., Rawiso M. and Francois B., Nature, 369 (1994) 387.

[10] Pitois O. and Francois B., Eur. Phys. J. B, 8 (1999) 225.

[11] Pitois O. and Francois B., Colloid Polym. Sci., 277 (1999) 574.

[12] Song L., PhD Thesis, Georgia Institute of Technology, 2005.

[13] Englert B. C., Scholz S., Leech P. J., Srinivasarao M. and Bunz U. H. F., Chem. Eur. J., 11 (2005) 995.
[14] Erdogan B., Song L. L., Wilson J. N., Park J. O., Srinivasarao M. and Bunz U. H. F., J. Am. Chem. Soc., 126 (2004) 3678.

[15] Song L., Bly R. K., Wilson J. N., Bakbak S., Park J. O., Srinivasarao M. and Bunz U. H. F., Adv. Mater., 16 (2004) 115.

[16] Weh L. and Venthur A., Macromol. Mater. Eng., 289 (2004) 227.

[17] Bormashenko E., Pogreb R., Stanevsky O., Bormashenko Y., Stein T., Gaisin V. Z., Cohen R. and Gendelman O. V., Macromol. Mater. Eng., 290 (2005) 114.

[18] Neitzel G. P. and Dell'Aversana P., Annu. Rev. Fluid Mech., 34 (2002) 267.

[19] Sharma V. and Srinivasarao M., in preparation.

[20] Meakin P., Rep. Prog. Phys., 55 (1992) 157.

[21] Crank J., The Mathematics of Diffusion, 2nd edition (Oxford University Press, New York) 1975.

[22] Hines A. J. and Maddox R. N., Mass Transfer: Fundamental and Applications (Prentice Hall, New Jersey) 1985.

[23] Cui L., Wang H. F., Ding Y. and Han Y. C., Polymer, 45 (2004) 8139.

[24] Maeda Y., Shimoi Y. and Ogino K., Polym. Bull., 53 (2005) 315.

[25] Doi M. and Edwards S. F., The Theory of Polymer Dynamics (Oxford University Press, Oxford) 1986.

[26] Steyer A., Guenoun P. and Beysens D., Phys. Rev. E, 48 (1993) 428.

[27] Peng J., Han Y. C., Yang Y. M. and Li B. Y., Polymer, 45 (2004) 447.

[28] Stenzel M. H., Barner-Kowollik C. and Davis T. P., J. Polym. Sci. Part A: Polym. Chem., 44 (2006) 2363.

[29] Vrentas J. S. and Vrentas C. M., J. Polym. Sci. Part B: Polym. Phys., 32 (1994) 187.

[30] Guerrier B., Bouchard C., Allain C. and Bernard C., AIChE J., 44 (1998) 791.

[31] Casper C. L., Stephens J. S., Tassi N. G., Chase D. B. and Rabolt J. F., Macromolecules, 37 (2004) 573.

[32] Hall D. B., Underhill P. and Torkelson J. M., Polym. Eng. Sci., 38 (1998) 2039.

[33] Bang J., Kim B. J., Stein G. E., Russell T. P., Li X., Wang J., Kramer E. J. and Hawker C. J., Macromolecules, 40 (2007) 7019. 